\title{
Phonon-plasmon coupling in Si doped GaN nanowires
}

\author{
E. Rozas-Jiménez ${ }^{\mathrm{a}}, \mathrm{A} . \mathrm{Cros}^{\mathrm{a}}$, S. Murcia-Mascarós ${ }^{\mathrm{a}}, \mathrm{Z}$. Fang ${ }^{\mathrm{b}, \mathrm{c}, \mathrm{d}}, \mathrm{B}$. \\ Daudin ${ }^{\mathrm{b}, \mathrm{c}}$ \\ ${ }^{a}$ Institute of Materials Science (ICMUV), University of Valencia, P.O. Box 22085, \\ ES-46071, Valencia, Spain \\ ${ }^{b}$ Univ. Grenoble Alpes, F-38000 Grenoble, France \\ ${ }^{c}$ CEA, INAC-SP2M, "Nanophysique et semiconducteurs" group, F-38000 Grenoble, \\ France \\ ${ }^{d}$ CNRS, Inst. NEEL, F-38042 Grenoble, France
}

\begin{abstract}
The vibrational properties of silicon doped GaN nanowires with diameters comprised between 40 and $100 \mathrm{~nm}$ are studied by Raman spectroscopy through excitation with two different wavelengths: 532 and $405 \mathrm{~nm}$. Excitation at $532 \mathrm{~nm}$ does not allow the observation of the coupled phonon-plasmon upper mode for the intentionally doped samples. Yet, excitation at $405 \mathrm{~nm}$ results in the appearance of a narrow peak at frequencies close to that of the uncoupled $\mathrm{A}_{1}(\mathrm{LO})$ mode for all samples. This behavior points to phonon-plasmon scattering mediated by large phonon wave-vector in these thin and highly doped nanowires.
\end{abstract}

Keywords: Raman scattering; nanowire; Gallium Nitride; Si doping; phonon-plasmon;

\section{Introduction}

Nanowires (NWs) are already established as important building blocks for the development of nanodevices, such as sensors [1], light emitting diodes [2], photovoltaic cells [3] or piezotronic devices [4], among others. In particular, GaN NWs present great advantages when compared to thin layers or quantum wells due to the strongly reduced density of extended defects. Of course, this increasing interest in NW devices requires a parallel effort in the development of new skills and techniques providing a reliable and statistically significant characterization of the nanostructures. For example doping 
of nanowires, a very important step in the development of these devices, requires the determination of carrier density, a task that is far from being standard in nanostructures. The nanoscale dimensions and the large NW aspect ratio render conventional transport measurements challenging and very time consuming due to the requirement of nanometer-size ohmic contacts. These difficulties are specially arduous when dealing with self organized NWs grown by molecular beam epitaxy (MBE), where typical dimensions do not exceed 2-3 $\mu \mathrm{m}$ in length and $20-200 \mathrm{~nm}$ in radius [5]. Taking into account these constraints, several optical tools not requiring special sample preparation, such as photoluminescence [6, 7] or Raman scattering [8, 9] have provided the determination of carrier concentration. Compared to photoluminescence, Raman scattering experiments are usually performed at room temperature and may as well deliver information about size, structure, local density, strain or crystallographic orientation [10]. The use of this technique for the study of NW doping is however often hindered by the difficult detection of the phonon-plasmon coupled modes $\left(\mathrm{LPP}^{+}, \mathrm{LPP}^{-}\right)$due to the presence of peaks from the substrate or to the over-damping of the plasmon modes [5, 9].

In this work we present a comparative study of the Raman spectra of self organized GaN NW ensembles highly doped with Si, obtained at two different excitation energies, namely 532 and $405 \mathrm{~nm}$. We will show that $405 \mathrm{~nm}$ excitation, though being still $400 \mathrm{meV}$ below the GaN band gap (3.06 eV vs. $3.45 \mathrm{eV}$ ), allows the observation of a narrow peak that arises at frequencies close to that of the uncoupled $\mathrm{A}_{1}(\mathrm{LO})$ mode, together with its overtone. The peak is ascribed to phonon-plasmon scattering that takes place for large phonon wave-vector.

\section{Samples and Experiment}

The GaN NWs studied here were grown by plasma-assisted MBE on Si (111) substrates. Details of the growth are given elsewhere [11, 12]. A Si effusion cell was used for n-type doping, performed by exposing GaN NWs to the Si flux [5]. The doping level was varied in the three samples studied by setting the $\mathrm{Si}$ cell temperature to 850,900 , and $938^{\circ} \mathrm{C}$, respectively. A not intentionally doped sample (NID) was also examined. The size of the NWs was investigated by scanning electron microscopy. The mean diameter was $45 \mathrm{~nm}$ for the NID sample and increased to $100 \mathrm{~nm}$ for the most doped sample. For the Raman experiments, a Horiba Jobin Yvon iHR320 spectrometer with Peltier-cooled charge coupled detector was used. Two different exci- 
tation wavelengths were employed: 532 and $405 \mathrm{~nm}$. The experiment was performed in backscattering geometry using a $50 \times$ microscope objective with 0.55 numerical aperture in order to focus the excitation laser along the c-axis of the as grown NWs. Raman spectra of the samples with different Si doping levels are shown in Figure 1 (a) for $532 \mathrm{~nm}$ excitation and (b) for $405 \mathrm{~nm}$ excitation. In both cases the bottom spectrum corresponds to the Si substrate, and is included as a reference. The $\mathrm{E}_{2 h}$ mode appears around 565.5 $\mathrm{cm}^{-1}$, right above the very strong $\mathrm{Si}$ peak from the substrate and slightly red-shifted from its relaxed value $\left(567 \mathrm{~cm}^{-1}\right)$ due to the small tensile strain induced by the incorporation of silicon in the lattice [5]. Indeed, the strong peak from the substrate hinders the observation of the $A_{1}(\mathrm{TO})$ mode or the $\mathrm{LPP}^{-}$. For the three samples with higher Si content a broad band centered at $658 \mathrm{~cm}^{-1}$ can be observed. It is ascribed to a disorder activated mode (DA) originated by phonon scattering with the increasing impurity content, and has been reported in samples doped with a variety of species [13, 14]. With $532 \mathrm{~nm}$ excitation, the $\mathrm{LPP}^{+}$mode is only observed for the NID sample, centered at $738 \mathrm{~cm}^{-1}$. This phonon-plasmon coupled mode arises as a small high energy shoulder of the surface optical (SO) mode [15]. For samples with higher doping levels the $\mathrm{LPP}^{+}$mode is overdamped and cannot be detected. The second order modes, on the contrary, can be observed for all samples. They appear above $1250 \mathrm{~cm}^{-1}$. Overtones and combination modes are present in this region, identified in order of increasing energy as a combination mode $\mathrm{TO}+\mathrm{LO}\left(\mathrm{A}_{1}\right.$ symmetry), a combination mode involving $\mathrm{SO}+\mathrm{LO}$, combination of $\mathrm{E}_{2 h}+\mathrm{LO}$, and an LO overtone. The relative intensity of the LO modes increases considerably when excited at $405 \mathrm{~nm}$ [Fig. 1 (b)], where both the first and second order modes can be easily detected for all samples. This is ascribed to the enhancement of the Fröhlich interaction when the excitation is close to a real electronic transition, an important mechanism in polar semiconductors, as already observed in GaN cubic layers [16]. In order to get insight into the evolution of the LO modes with doping, a multi-Lorentzian fit has been performed to the spectra. The center of the first order $\mathrm{A}_{1}(\mathrm{LO})$ mode and that of its overtone divided by two are plotted in Figure 2 as a function of the Si cell temperature used for doping. The frequency of the overtone divided by two is lower than that of the first order mode, a characteristic expected from the fact that the second order process may involve phonons out of the center of the Brillouin zone. Besides this effect, the modes present a clear decrease in frequency (almost $6 \mathrm{~cm}^{-1}$ ) as the Si content in the samples increases. The shift is too large to be due to 
the small tensile strain in the samples. Furthermore, the usual Drude model for the phonon-plasmon coupling predicts a blue-shift of the coupled LPP ${ }^{+}$ mode for increasing carrier concentration [17], opposite to the one observed here. Actually, the electrical characteristics of these NWs have been studied in detail in Ref. [5], where it was shown that they present high carrier densities of the order of $5 \times 10^{18} \mathrm{~cm}^{-3}$ and larger. The Drude model for the LPP modes would then result in a broad coupled phonon-plasmon mode above $900 \mathrm{~cm}^{-1}$, that is not observed in our samples. As we will show below, the observed red-shift of the mode for highly doped samples, together with the absence of the $\mathrm{LPP}^{+}$for large wavelength excitation, is the experimental footprint of scattering mediated by large phonon wave-vector $[16,18]$.

\section{Discussion}

As a first step for understanding the evolution of the phonon modes with doping in the case of large plasmon damping $\Gamma$, we have calculated the dispersion of the LPP modes as a function of the plasmon frequency for different $\Gamma$ at zero phonon wave-vector $(q=0)$. Let us recall that $\Gamma$ is inversely proportional to the carrier mobility, that is expected to decrease in highly doped samples. The calculations are based on the formalism developed by Hon and Faust $[19,20]$ for the phonon-plasmon coupled modes. The Faust-Henry coefficient for GaN needed for the calculation has been recently determined by Irmer et al. [21] to be -3.46 . The results of the model are represented in Figure 3 for values of the plasmon damping $\Gamma$ ranging from 100 to 2000 $\mathrm{cm}^{-1}$ (carrier mobilities from $\sim 470$ to $25 \mathrm{~cm}^{2} \mathrm{~V}^{-1} \mathrm{~s}^{-2}$ ). For the calculation a constant phonon damping of $\gamma=10 \mathrm{~cm}^{-1}$ was considered. This value corresponds to the full width at half maximum (FWHM) of the uncoupled $\mathrm{A}_{1}(\mathrm{LO})$ peak, and is characteristic of GaN doped layers [17]. In our samples the FWHM, extracted from a Lorentzian fit to the observed peak, is somewhat larger $\left(14-25 \mathrm{~cm}^{-1}\right)$. However, we have checked that the calculated LPP frequencies depend very weakly on the value of the phonon damping provided it is much smaller than the plasmon damping, which is the case in our samples. For small plasmon damping ( $\Gamma$ below $800 \mathrm{~cm}^{-1}$, i.e. mobilities larger than $60 \mathrm{~cm}^{2} \mathrm{~V}^{-1} \mathrm{~s}^{-2}$ ), the usual two coupled LO-phonon plasmon modes of the Drude model can be observed, classified as upper $\left(\mathrm{LPP}^{+}\right)$and lower $\left(\mathrm{LPP}^{-}\right)$branches. However, as soon as the plasmon damping exceeds this value, this separation lacks of physical meaning, and only one branch is

observed. For small enough carrier density (lower than $7 \times 10^{18} \mathrm{~cm}^{-3}$ in the 
figure), one of the modes has a phonon-like character, with energies close to that of the LO phonon. For higher carrier densities the electric field responsible of the LO-TO splitting is screened by the free carriers, and the phonon-like mode decreases its frequency, adopting energies close to that of the TO. The other mode has plasmon-like character and is over-damped. Since the electrical properties of the NID sample were studied in detail [5], we know that most of its NWs present mobilities higher than $100 \mathrm{~cm}^{2} \mathrm{~V}^{-1} \mathrm{~S}^{-1}$. We expect then the $\mathrm{LPP}^{+}$to appear at frequencies higher than that of the uncoupled $\mathrm{A}_{1}(\mathrm{LO})$, a behavior that is indeed observed for the NID sample. However, in the case of the intentionally doped NWs the mode emerges as a narrow peak below the uncoupled $\mathrm{A}_{1}(\mathrm{LO})$. If the red-shift were due to large plasmon damping, a much broader peak would be expected. Actually, the appearance of a well defined peak at frequencies below that of the $\mathrm{A}_{1}(\mathrm{LO})$ is expected when first order Raman scattering is mediated by large phonon wave-vectors [22]. A similar behavior has already been reported for strongly doped InN [18] and cubic GaN [16]. Scattering mediated by large wave-vectors allows the observation of a relatively narrow peak below the uncoupled LO mode in samples with large carrier density. This mode is not expected to change significantly for increasing doping, and can be promoted by excitation close to the band-gap [16]. The value of the phonon wave-vector required for this scattering needs to be larger than the Thomas-Fermi wave-vector [22], that is, of the order of $10^{7} \mathrm{~cm}^{-1}$. The source of these large $q$ values is not clear, but we have to take into account that the structural disorder promoted by the incorporation of Si impurities in the NWs shows up as a widening of the $\mathrm{E}_{2 h}$ mode, that increases to $30 \mathrm{~cm}^{-1}$ for the most doped sample. Consequently, phonon scattering by charged impurities, together with scattering mediated by the NW surface of these thin NWs are the most probable wave-vector sources.

The evolution of the phonon-plasmon coupled modes in the case of large wave-vector scattering can be accounted for through the Lindhard-Mermin model $[16,23]$. This model gives a complete description of the plasma behavior in polar semiconductors as function of carrier density, plasmon damping and phonon wave-vector. Figure 4 gives the evolution of the phonon-plasmon coupled mode as a function of carrier concentration considering a plasmon damping of $500 \mathrm{~cm}^{-1}$ (mobility $\sim 95 \mathrm{~cm}^{2} \mathrm{~V}^{-1} \mathrm{~s}^{-1}$ ). The calculations have been performed at 0 Kelvin and for three wave-vector values. They are expressed as multiples of $q_{0}=7 \times 10^{5} \mathrm{~cm}^{-1}$ that corresponds to the phonon wave-vector expected for backscattering excited at $405 \mathrm{~nm}$. For high carrier 
densities the phonon is red-shifted, following a similar trend as observed in our samples.

\section{Summary and Conclusions}

We have compared the Raman spectra of GaN NWs obtained at two different excitation frequencies: 532 and $405 \mathrm{~nm}$. In both cases the $\mathrm{A}_{1}(\mathrm{TO})$ mode is hidden by the strong peak of the Si substrate, and cannot be detected. Excitation with $405 \mathrm{~nm}$ allows the observation of a peak close to the frequency of the uncoupled $\mathrm{A}_{1}(\mathrm{LO})$ mode in all the samples, that shifts to lower frequencies with increasing $\mathrm{Si}$ content. Meanwhile, excitation at $532 \mathrm{~nm}$ does not provide information about the $L O$ mode for the intentionally doped samples. This behavior indicates that the scattering is mediated by large values of the phonon wave-vector that could have its origin in the phonon interaction with charged impurities and scattering by the NW surface. The red-shift of the phonon-plasmon coupled mode can be accounted for through the Lindhard-Mermin model with wave-vector values of the order of $10^{7} \mathrm{~cm}^{-1}, i$. e., of the order of the Thomas-Fermi wave-vector.

Acknowledgements The authors acknowledge Julien Pernot for a critical reading of the manuscript. Partial finantial support from HAR201452671, MEC (Spain) is also acknowledged.

[1] X. Chen, C. K. Y. Wong, C. A. Yuan, and G. Zhang, Sensors and Actuators B 177, 178 (2013).

[2] X. Dai, A. Messanvi, H. Zhang, Ch. Durand, J. Eymery, C. Bougerol, F. H. Julien, and Maria Tchernycheva, Nano Lett. 15, 6958 (2015).

[3] B. Tian, X. Zheng, T. J. Kempa, Y. Fang, N. Yu, G. Yu, J. Huang, and C. M. Lieber, Nature 449, 885 (2007).

[4] Z. L. Wang, Nano Today 5, 540 (2010).

[5] Z. Fang, E. Robin, E. Rozas-Jiménez, A. Cros, F. Donatini, N. Mollard, J. Pernot, and B. Daudin, Nano Lett. 15, 6794 (2015).

[6] T. Stoica, R. J. Meijers, R. Calarco, T. Richter, E. Sutter and H. Lüth Nano Lett. 61541 (2006). 
[7] D. Lindgren, O. Hultin, M. Heurlin, K. Storm, M. T. Borgström, L. Samuelson, and A. Gustafsson, Nanotech. 26045705 (2015).

[8] K. Jeganathan, R. K. Debnath, R. Meijers, T. Stoica, R. Calarco, D. Grtzmacher, and H. Lüth, Appl. Phys. Lett. 105, 123707 (2009).

[9] P. Tchoulfian, F. Donatini, F. Levy, B. Amstatt, A. Dussaigne, P. Ferret, E. Bustarret, and J. Pernot, Appl. Phys. Lett. 103, 202101 (2013).

[10] A. Cros, Phys. Stat. Sol. RRL 7, 727738 (2013).

[11] O. Landre, R. Songmuang, J. Renard, E. Bellet-Amalric, H. Renevier, and B. Daudin, Appl. Phys. Lett. 93, 183109 (2008).

[12] R. Mata, K. Hestroffer, J. Budagosky, A. Cros, C. Bougerol, H. Renevier, and B. Daudin, J. Cryst. Growth 334, 177 (2011).

[13] R. Cuscó, L. Artús, D. Pastor, F. B. Naranjo, and E. Calleja, Appl. Phys. Lett. 84, 897 (2004).

[14] S. Fritze, A. Dadgar, H. Witte, M. Bügler, A. Rohrbeck, J. Bläsing, A. Hoffmann, and A. Krost, Appl. Phys. Lett. 100, 122104 (2012).

[15] R. Mata, A. Cros, K. Hestroffer, and B. Daudin, Phys. Rev. B 85, 035322 (2012).

[16] M. Ramsteiner, O. Brandt, and K. H. Ploog, Phys. Rev. B 58, 1118 (1998).

[17] T. Kozawa, T. Kachi, H. Kano, Y. Taga, M. Hashimoto, N. Koide, and K. Manabe, J. Appl. Phys 75, 1098 (1994).

[18] F. Demangeot, C. Pinquier, J. Frandon, M. Gaio, O. Briot, B. Maleyre, S. Ruffenach, and B. Gil, Phys. Rev. B 71, 104305 (2005).

[19] D. T. Hon and W. L. Faust, Appl. Phys. 1, 241, 1973.

[20] G. Irmer, M. Wenzel, and J. Monecke, Phys. Rev. B 56, 9524 (1997).

[21] G. Irmer, C. Röder, C. Himcinschi, and J. Kortus, J. Appl. Phys 116, 245702 (2014). 
[22] Y.-J. Cho, M. Ramsteiner, and O. Brandt, Phys. Rev. B 85, 195209 (2012).

[23] G. Grosso and G. Pastori Parravicini, in Solid State Physics, Second Edition, page 324. Ed. Elsevier, Academic Press (2014). 


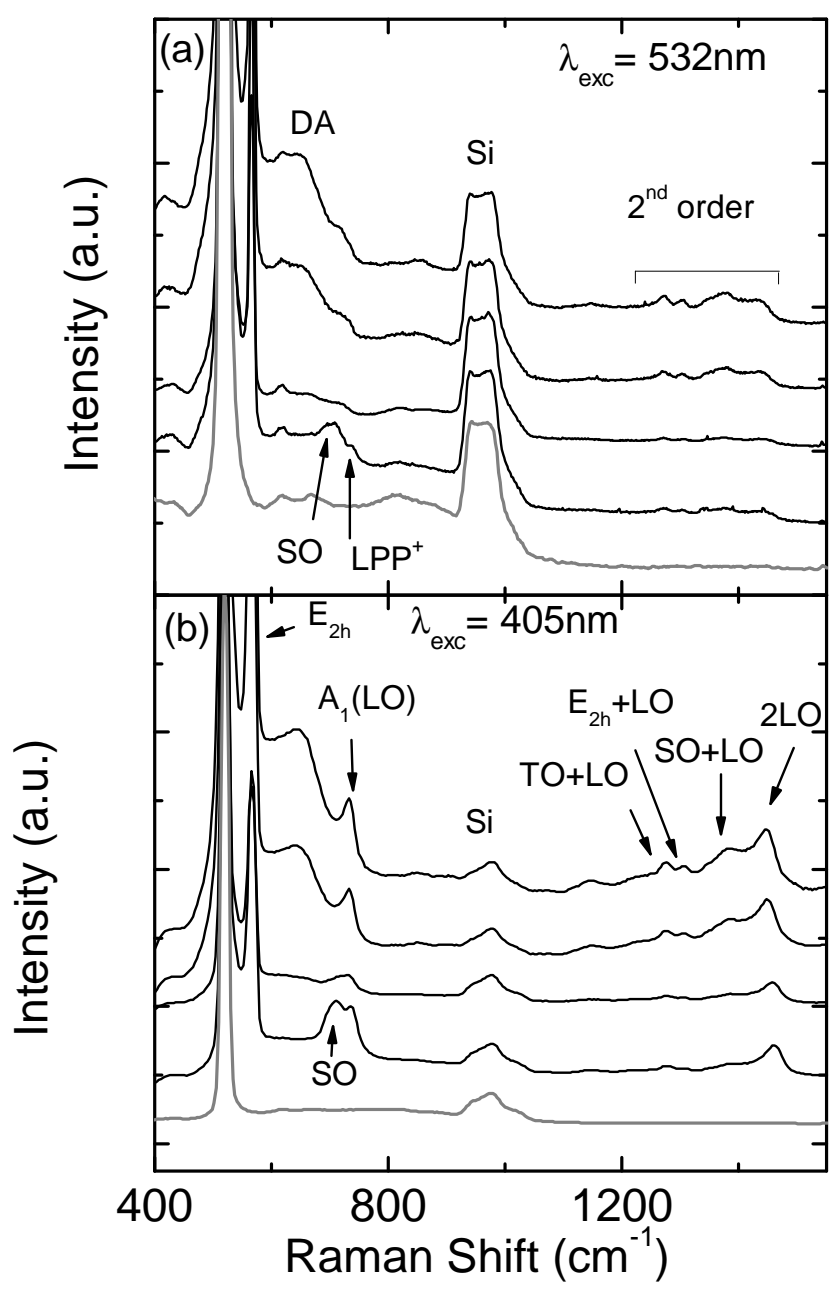

Figure 1: Raman spectra obtained with (a) $532 \mathrm{~nm}$ excitation and (b) $405 \mathrm{~nm}$ excitation. From bottom to top the spectra in each figure correspond to the Si reference, NID, and samples doped by setting the Si cell temperature to 850,900 and $938^{\circ} \mathrm{C}$. 


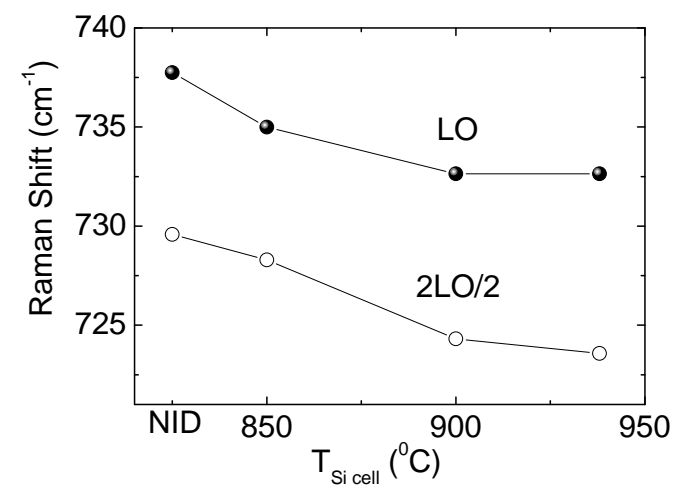

Figure 2: Evolution of the first (black dots) and second order (empty dots) LO modes with the temperature of the silicon cell used for doping during growth. The frequency of the second order LO mode has been divided by two for comparison. The lines are guides to the eyes.

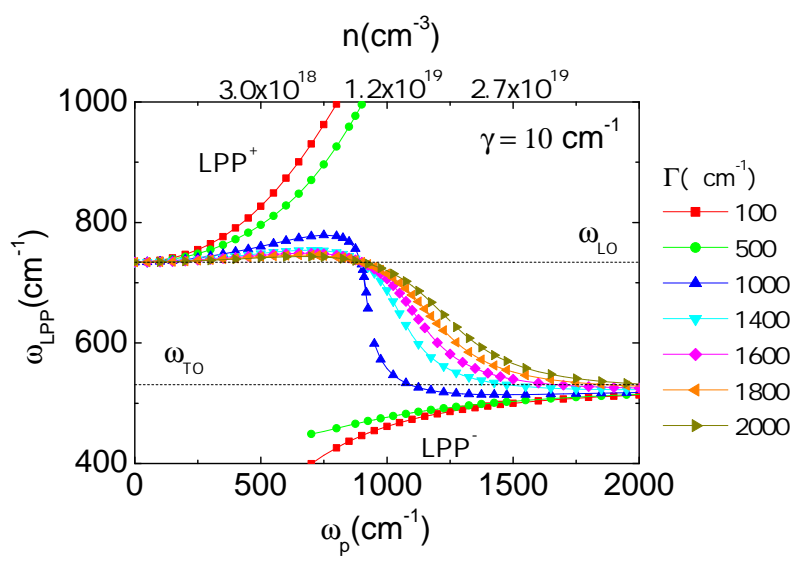

Figure 3: Dependence of the frequency of the phonon-plasmon coupled modes with plasma frequency and carrier concentration for different values of the plasmon damping. The full width at half maximum of the $\mathrm{A}_{1}(\mathrm{LO})$ mode $(\gamma)$ has been set to $10 \mathrm{~cm}^{-1}$. 


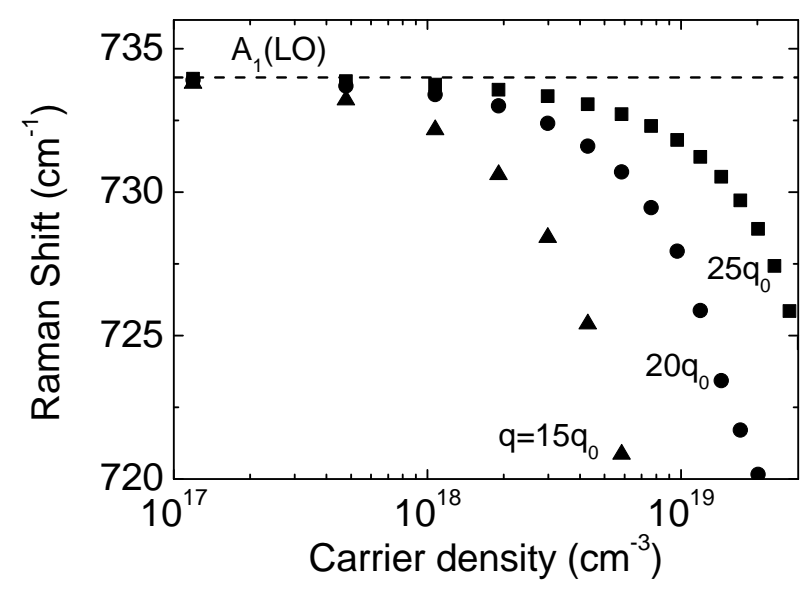

Figure 4: Frequency of the phonon-plasmon coupled modes as a function of carrier concentration in the case of large wave-vector scattering based on the Lindhard-Mermin model. 